\title{
Conference Paper Bragg Fibers for Absorption-Based Sensing
}

\author{
Vlastimil Matějec, Ivan Kasik, Ondrej Podrazky, and Ivo Barton \\ Institute of Photonics and Electronics AS CR, v.v.i., Chaberská 57, 18251 Prague 8, Czech Republic \\ Correspondence should be addressed to Vlastimil Matějec; matejec@ufe.cz
}

Received 12 December 2014; Revised 18 March 2015; Accepted 19 March 2015

Academic Editor: Yoshihiko Takeda

This Conference Paper is based on a presentation given by Vlastimil Matějec at "Meeting on Materials and Their Applications for Devices and Sensors 2014" held from 5 November 2014 to 7 November 2014 in Mahdia, Tunisia.

Copyright (C) 2015 Vlastimil Matějec et al. This is an open access article distributed under the Creative Commons Attribution License, which permits unrestricted use, distribution, and reproduction in any medium, provided the original work is properly cited.

\begin{abstract}
Photonic band gap Bragg fibers can be employed for delivery of high powers in medicine, lighting and heating systems, electricity production, and the development of optical sensors of physical and chemical variables. In this paper, results of testing Bragg fibers with hollow cores for $\mathrm{pH}$ detection based on absorbance measurements on solutions filled in the cores. Bragg fibers with claddings of three pairs of high- and low-index layers and hollow cores of $70 \mu \mathrm{m}$ in diameter were fabricated. Photonic band gap bands of the fibers were estimated theoretically. Aqueous solutions of methyl orange (MO) and hydrochloric acid and bromothymol blue (BTB) in Sörensen buffers were filled into the cores and spectra of $\mathrm{MO}$ and BTB were measured for different $\mathrm{pH}$. Calibration curves typical for optical pH sensors were derived from the measured spectra with a reproducibility of about $10 \%$.
\end{abstract}

\section{Introduction}

Recently, optical fibers have shown their performance for telecommunications and lasers [1] as well as for sensing of physical and chemical variables [2-11]. Different types of sensing platforms have been investigated including evanescent-wave and reflection sensors, sensors based on fiber-optic tapers, and Bragg or long-period fiber gratings inscribed in fibers. Fiber-optic sensors often employ fibers with the core refractive indices higher than those of the cladding. Such fibers include telecommunication types of silica fibers, that is, polymer-clad silica and multimode and single-mode fibers which, however, have to be modified, for example, by optical gratings, thin layers, and so forth $[2-4,6]$, to increase their sensitivity to external variables such as temperature, pressure, mechanical deformations, and chemicals. Special sensing fibers such as D-fibers, etchedfibers, fiber tapers, and surface plasmon resonance (SPR) fiber-optic sensors $[2,5-7]$ have also been developed which exhibit improved sensing performance. Recently, special types of optical fibers relying on novel physical principles such as microstructured fibers, photonic crystal fibers [8, 9], and photonic band gap Bragg fibers [10-14] have been investigated for physical and chemical sensing.
Photonic crystal fibers (PCFs) consist of a solid or air core surrounded by a periodic array of microscopic air holes. Microstructured fibers (MSFs) are prepared with solid cores and air-hole arrays do not exhibit any periodicity. There two distinct guiding mechanisms in PCFs [9]. First one is based on the modified total light reflection on a cladding composed of solid material whose mean refractive-index is decreased due to air holes in it. This mechanism takes place at PCFs with solid cores and in MSFs. In PCFs with air cores, light is confined and guided in the core due to photonic band gap of a periodic array of air holes around the core. PCFs and MSFs have been fabricated from silica, chalcogenide glasses, polymers, and so forth.

Photonic band gap guiding mechanism takes also place at photonic band gap Bragg fibers (PBFs) $[9,10]$. Such fibers consist of solid or air cores surrounded by Bragg claddings of several pairs of Bragg layers, one of a high refractive index and one of a low refractive index (see a schematic refractiveindex profile in Figure 1). Bragg fibers have been fabricated from doped silica glasses, chalcogenide glasses, or polymers.

Waveguiding characteristics of PCFs can be controlled by dimensions of air holes and core, by distances of air holes, by fiber lengths, and so forth $[8,9]$. This control 
represents the important advantage of PCFs in comparison with standard multimode or single-mode fibers and it can be employed in PCF sensors. A number of physical and chemical sensors based on PCFs have been investigated as one can find in several reviews on PCF sensors $[8,9]$. Physical PCF sensors for monitoring strains, temperature, pressure, and so forth have been investigated. PCF sensors based on the refractive-index, absorbance, or luminescence detection have been developed and tested for the detection of gaseous or liquid chemicals filled in hollow fiber cores.

PCFs offer unique advantages for chemical sensors and biosensors. As their air holes are running along its length, such a fiber can contain biological and chemical analytes in in the close vicinity of the fiber core or inside the core. The length of fibers and thus the detection length can be controlled from several centimeters to meters. PCFs can be used simultaneously for light guiding and as a fluidic channel. Such channels can be further functionalized with transducing layers. As the PCF core and air holes have small sizes a very small analyte volume is required for sensing. Using PCFs analyte volumes on a level of microliters can be used while in conventional optics measurement techniques minimum volumes of hundred microliters are used [9]. Moreover, walls of the hollow core and air holes of PCFs can be modified by detection layers which enable further advancing detection performance of PCFs. PCF core and hole walls can also be modified with thin metallic layers of silver or gold and can be used in SPR sensors and biosensors. Such fibers have successfully been employed in label-free biosensors.

Photonic band gap Bragg fibers (PBFs) can offer similar advantages for sensing applications as PCFs fibers, that is, increased detection sensitivities, long detection lengths, small detection volumes, and so forth. However, in PBFs only the hollow core can be used for detection of chemicals. In several papers, the performance of PBFs for sensing has been investigated [10-14]. Effects of changes of the complex refractive index of material in a PBF hollow core have been theoretically examined [10]. It has been shown that band gap fiber characteristics are very sensitive to changes of the real part of the refractive index in the hollow core. Results of such calculations have been implemented into the development of an experimental PBF refractometer based on a PBF with a Bragg cladding layers fabricated from polymethylmethacrylate and polystyrene [11-13]. Similar PBFs have been tested for microdisplacement sensing [14].

This paper presents results of the experimental investigation of the performance of silica photonic band gap Bragg fibers for $\mathrm{pH}$ detection based on absorption $\mathrm{pH}$ indicators such as methyl orange and bromothymol blue. Spectral responses of the fibers filled in the hollow fiber cores with aqueous indicator solutions are presented as well as calibration curves showing the performance of such fibers for absorption-based $\mathrm{pH}$ sensing.

\section{Experimental}

2.1. Preparation of Bragg Fibers. Bragg fibers with air cores and Bragg claddings consisting of three pairs of high- and

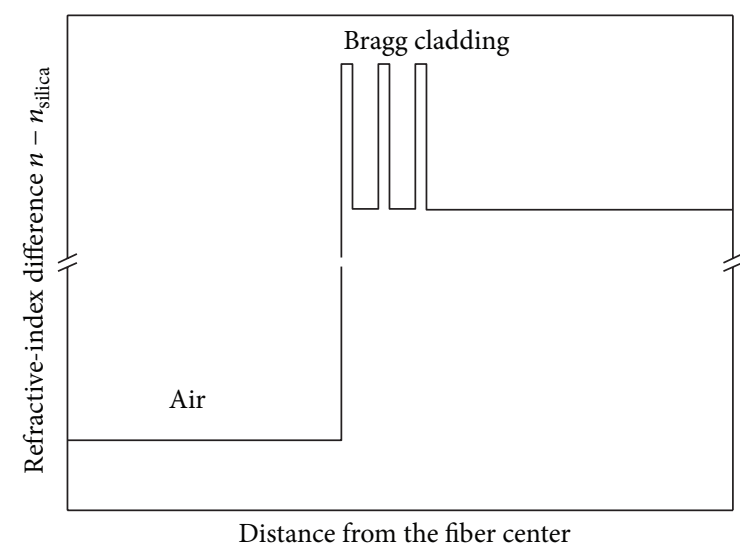

FIGURE 1: Schematic refractive-index profile of Bragg fiber.

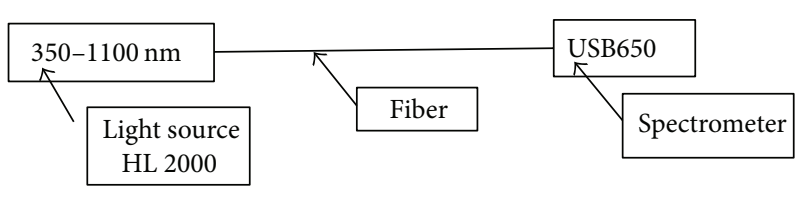

FIGURE 2: Scheme of the experimental setup.

low-index layers (see the schematic refractive-index profile in Figure 1) $[15,16]$ were prepared for testing in sensing experiments. Preforms for drawing of such Bragg fibers were fabricated by the MCVD method [15]. At experiments, several thin layers of silica slightly doped with fluorine were deposited onto the inner wall of a high-quality silica substrate tube at first. Then, the high-index Bragg layer was prepared from silica doped with germanium dioxide and phosphorous pentoxide. The following low-index Bragg layer was composed of silica doped slightly with phosphorous pentoxide. The same procedure of preparing the high- and low-index layers was repeated in order to fabricate three pairs of Bragg layers.

Bragg fibers were drawn from tubes with applied layers, the preforms, using a graphite resistance furnace Centorr (USA). Temperatures slightly below $2000^{\circ} \mathrm{C}$ were used for drawing fibers with air cores (see Figure 1). By increasing temperatures above $2000^{\circ} \mathrm{C}$ the full collapse of the preform in a hot zone of the furnace took place. Fibers with a glass diameter of $125 \mu \mathrm{m}$ were prepared from the preforms. They were coated with a protective polymeric jacket of UV-curable acrylate (De Sotto).

2.2. Cross-Sections of Prepared Fibers. Cross-sections and dimensions of prepared Bragg fibers were characterized by optical microscopy in the transmission mode. Fiber segments with a length up to $2 \mathrm{~cm}$ were used in these measurements. The segments were cut off from prepared fibers by using a knife of hard metal without removing the polymeric jacket.

2.3. Characterization of Sensing Fibers. Transmission spectra of prepared Bragg fibers with hollow cores filled in with tested aqueous solutions were measured by using a setup 
schematically shown in Figure 2 . The setup consisted of a halogen lamp Ocean Optics HL 2000 as a light source and an Ocean Optics USB 650 diode array spectrometer as a detector. Tested fiber segments with lengths of $15 \mathrm{~cm}$ were joined to the source and detector via FC connectors.

Immersing solutions of $\mathrm{pH}$ indicators methyl orange (MO) dissolved in hydrochloric acid and water in concentrations from $10^{-2}$ to $10^{-4} \mathrm{~mol} / \mathrm{L}$ and bromothymol blue (BTB) in Sörensen buffer solutions mixed from sodium and potassium phosphates were used for testing spectral absorption responses of prepared Bragg fibers. The concentration of both indicators of $0.01 \mathrm{~g} / \mathrm{L}$ was used in all immersing solutions. All the chemicals were supplied by Sigma-Aldrich, CR.

In experiments each immersing solution was filled into the hollow cores due to the effect of capillary elevation. For such purpose the input end of the fiber was immersed in the tested solution for $10 \mathrm{~s}$. A solution column with a length of about $10 \mathrm{~cm}$ was formed in the capillary. After measurements, the solution was removed from the core by using the overpressure of about 5 bar. Then, the hollow core was rinsed out by distilled water for three times. During these operations the tested fiber was removed from the setup, together with both connectors. Such measurements were repeated for three times.

\section{Results and Discussion}

Bragg fibers tested for absorption-based sensing were prepared by the MCVD method. Fibers with similar Bragg claddings have successfully been tested for delivery of high laser powers at $1064 \mathrm{~nm}$ [16]. In this paper, Bragg fibers with hollow-core diameters of about $70 \mu \mathrm{m}$ were used.

A photo of the cross-section of the fiber determined by optical microscopy is shown in Figure 3(a). As the microscope resolution did not allow us to determine directly dimensions of high- and low-index layers from the photo, the following procedure has been used for such determination. In the procedure, a fiber without any hollow core was prepared from the same perform as that used for the fiber with the hollow core (see the cross-section in Figure 3(b)). Then, dimensions of the high- and low-index layers in Figure 3(a) were calculated from those in Figure 3(b) by using the massconservation law. These dimensions are approximately of $0.3 \mu \mathrm{m}$ for the high-index layers and of $0.7 \mu \mathrm{m}$ for the low-index ones. A refractive-index difference of about 0.03 between the high- and low-index layers was assumed on the basis of results reported elsewhere [15].

Photonic band gap characteristics of prepared Bragg fibers have been estimated theoretically. Such estimations employed a planar approximation of the fiber structure (the core diameter $\gg$ wavelength) and the theory of stratified media [17]. Band gap edges, that is, values of effective indices between which band gap condition is satisfied, were calculated from

$$
\left|\cos \left(k_{2} d_{2}\right) \cos \left(k_{1} d_{1}\right)-\delta \sin \left(k_{2} d_{2}\right) \sin \left(k_{1} d_{1}\right)\right|=1
$$

$$
\begin{array}{r}
\delta=\frac{1}{2}\left(\frac{k_{1}}{k_{2}}+\frac{k_{2}}{k_{1}}\right) \\
k_{i}=\frac{2 \pi}{\lambda} \sqrt{n_{i}^{2}-n_{\mathrm{eff}}^{2}} \quad i=1,2 .
\end{array}
$$

The effective indices $n_{\text {eff }}$ were determined for TE modes only because they overlap band gaps of TM modes [17] for wavelengths 500, 550, 600, 650, and $700 \mathrm{~nm}$ taking into account the dimensions and refractive-index difference of the Bragg layers discussed above. A refractive index of 1.33, corresponding to water-filled hollow cores, was used as the core refractive index. Results are presented in Figure 4. Black rectangles describe ranges of effective indices of TE optical modes propagating in the core.

Results in Figure 4 show that there are optical modes confined in the core which can be used for the detection of absorbance changes in the core. In addition to these modes, there are modes with effective refractive indices close to 1.33 corresponding to paraxial rays that can also propagate in the liquid-filled core of straight fibers. Finally, evanescent waves of modes propagating in first high-index cladding layer can also contribute to the sensitivity of tested Bragg fibers to absorption changes in the cores.

An example of measured spectral responses of one Bragg fiber of bromothymol blue in Sörensen buffer solutions for different $\mathrm{pH}$ is shown in Figure 5.

A calibration curve determined from measured spectral responses for different $\mathrm{pH}$ of bromothymol blue solutions and for a wavelength of $610 \mathrm{~nm}$ is shown in Figure 6. The curve is typical for $\mathrm{pH}$ measurements. However, error bars in Figure 6 show that results determined with the same fiber but at different experiments have the reproducibility of about $10 \%$. Such results can probably be explained by changes of launching conditions during the exchange of measured samples and by different lengths of sample columns in the hollow cores filled-in by the capillary elevation.

Experimental data in Figure 6 were fitted with a Boltzmann plot (see the curve in Figure 6) [18]. A detection sensitivity of about $0.751 / \mathrm{pH}$ unit was determined from the plot for $\mathrm{pH}$ values in a linear range from 6.5 to 7.5. From the sensitivity a resolution of about $0.025 \mathrm{pH}$ unit was determined for a single measurement in the linear range. This value is by about two times higher than that reported for a commercial fiber-optic $\mathrm{pH}$ sensor PreSens [19].

A typical spectral response of a Bragg fiber to methyl orange solutions in hydrochloric acid of different $\mathrm{pH}$ is shown in Figure 7. The corresponding calibration curve is shown in Figure 8. Similarly as for the bromothymol blue solutions a measurement reproducibility of about $10 \%$ was also determined for methyl orange.

From the Boltzmann plot in Figure 8, a sensitivity value of about $0.81 / \mathrm{pH}$ unit was determined in a linear $\mathrm{pH}$ range from 3.2 to 4.5 . This sensitivity corresponds to a resolution of about $0.02 \mathrm{pH}$ unit for a single measurement in which the absorbance can be determined with a precision up to $3 \%$. However, for repeated measurements the resolution 


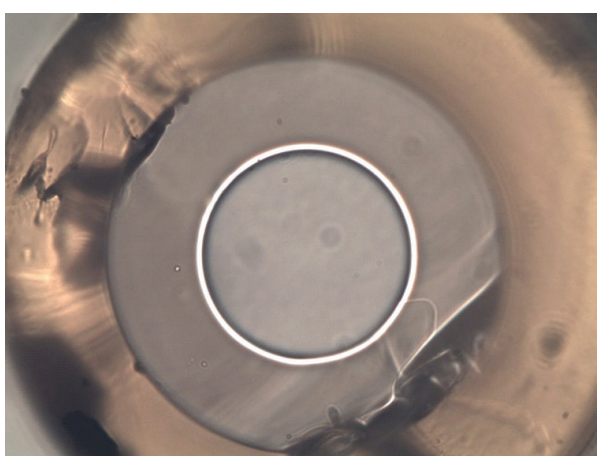

(a)

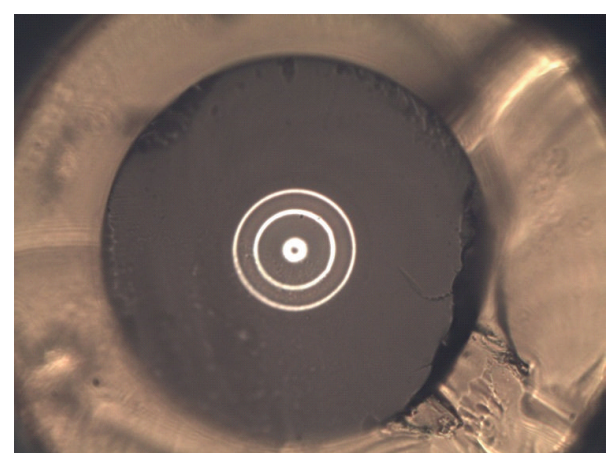

(b)

Figure 3: Cross-sections of prepared fibers; (a) Bragg fiber with air-core diameter of $70 \mu \mathrm{m}$; (b) fiber without air core fabricated by full collapsing of the preform.

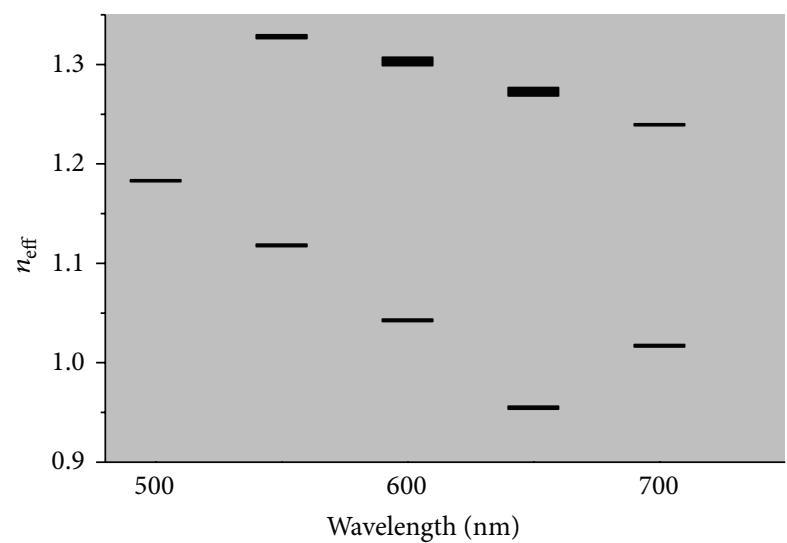

Photonic band gap

Figure 4: Calculated edges of photonic band gap bands for TE modes.

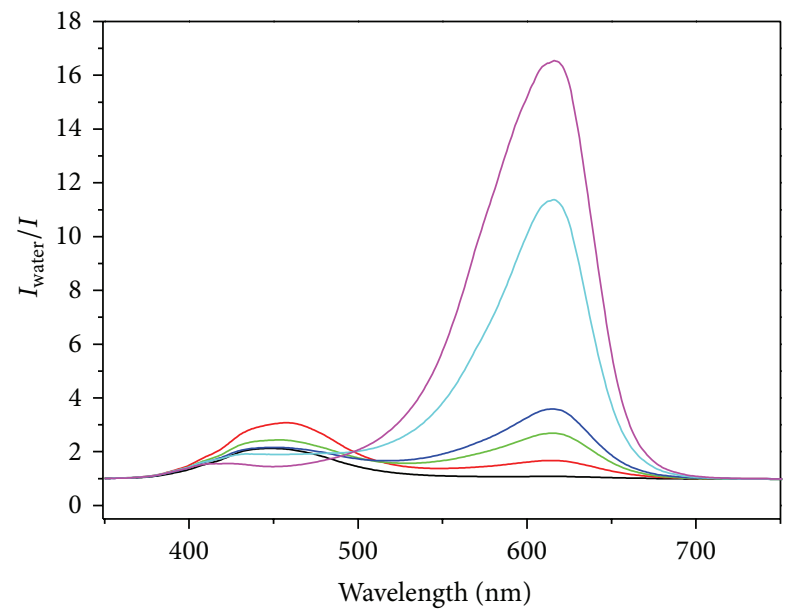

$$
\begin{aligned}
-\mathrm{pH}=5.59 & -\mathrm{pH}=6.98 \\
-\mathrm{pH}=6.24 & -\mathrm{pH}=7.38 \\
\mathrm{pH}=6.64 & -\mathrm{pH}=8.04
\end{aligned}
$$

FIGURE 5: Spectral responses of the Bragg fiber to solutions of bromothymol blue in Sörensen buffers with different $\mathrm{pH}$.

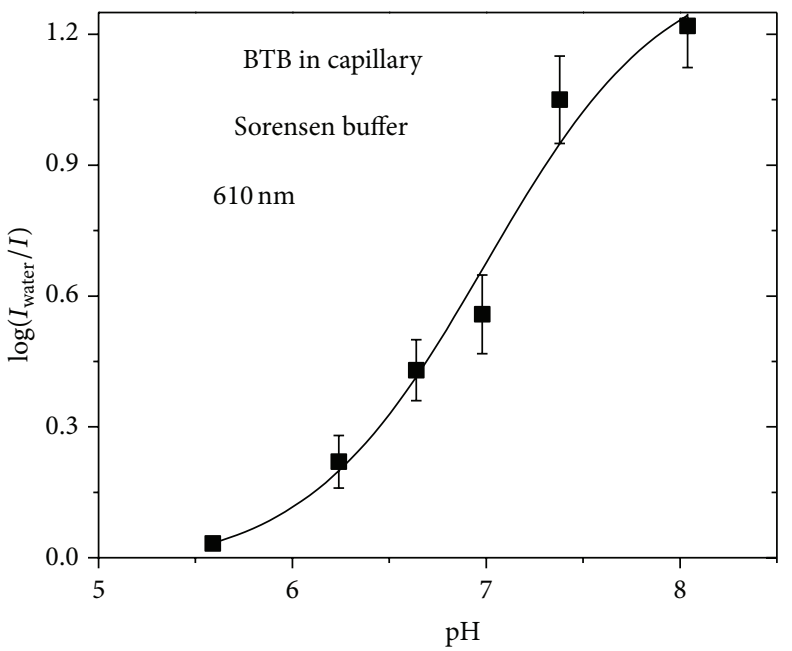

Figure 6: Calibration curve of the Bragg fiber for $\mathrm{pH}$ detection by using bromothymol blue.

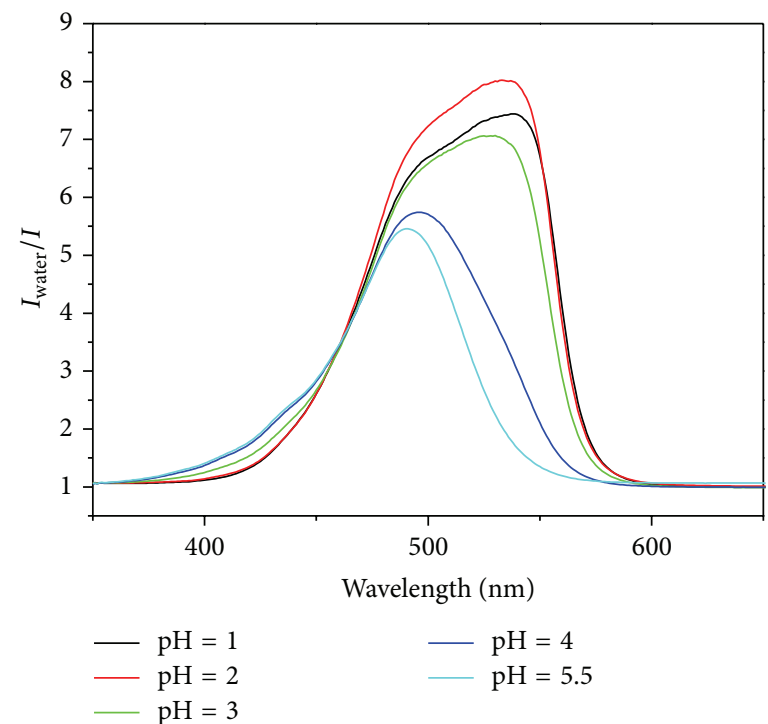

FIGURE 7: Spectral responses of the Bragg fiber to methyl orange in solutions of hydrochloric acid with different $\mathrm{pH}$. 


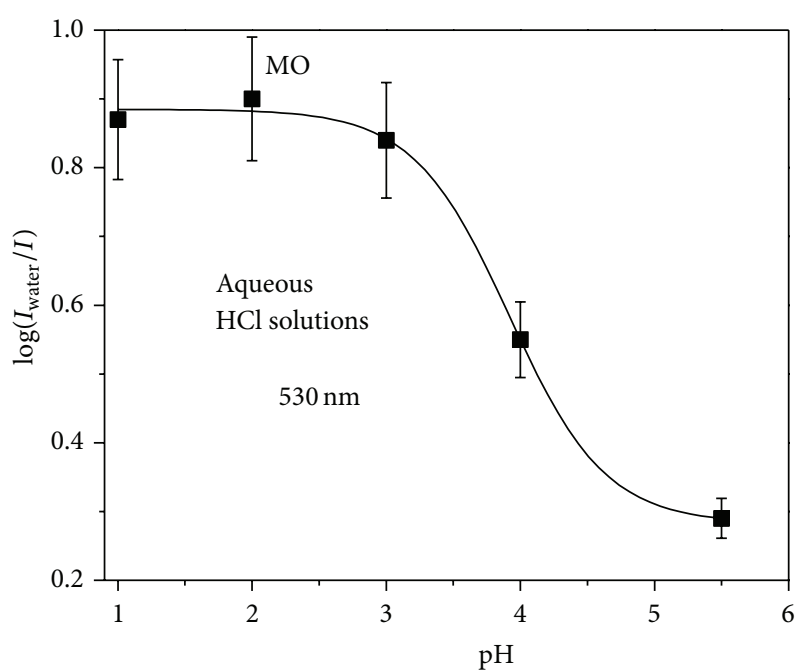

Figure 8: Calibration curve of the Bragg fiber for $\mathrm{pH}$ detection by using methyl orange.

decreases to about $0.06 \mathrm{pH}$ unit because in this case the reproducibility is of about 10\% (see the error bars in Figure 8).

Although the investigated sensing element will probably not compete with commercially available $\mathrm{pH}$ sensors [19], it could be useful for spectral measurements in weakly absorbing gases and liquids because it offer long detection lengths and small detection volumes. However, for practical sensing, the measurement reproducibility has to be improved which is critically dependent on the amount of analyte in the fiber hole. This can be achieved, for example, by using a controlled vacuum pump. The filling-in and removing tested samples from the core without changing launching condition can also improve the reproducibility [20]. The sensing performance of such an element can be further extended by applying suitable detection layers onto the wall of the hollow fiber core, for example, by using the sol-gel method. However, changes of waveguiding characteristics of sensing fibers due to such layers can be expected.

\section{Conclusions}

It has been shown that Bragg fibers with hollow cores can be used for the detection of absorbance changes in the cores and therefore for $\mathrm{pH}$ detection by using colorimetric $\mathrm{pH}$ indicators. Such sensing fibers offer us long detection paths and small volumes of detected samples. However, several technical issues related mainly to filling in tested samples into the cores are necessary to be solved to improve the measurement reproducibility that is so far on a level of $10 \%$.

\section{Conflict of Interests}

The authors declare that there is no conflict of interests regarding the publication of this paper.

\section{Acknowledgment}

This work was financially supported by the Czech Science Foundation (Contract P102/12/2361).

\section{References}

[1] E. Desurvire, C. Kazmierski, F. Lelarge et al., "Science and technology challenges in XXIst century optical communications," Comptes Rendus Physique, vol. 12, no. 4, pp. 387-416, 2011.

[2] O. S. Wolfbeis, "Fiber-optic chemical sensors and biosensors," Analytical Chemistry, vol. 80, no. 12, pp. 4269-4283, 2008.

[3] L. Mescia and F. Prudenzano, "Advances on optical fiber sensors," Fibers, vol. 2, no. 1, pp. 1-23, 2014.

[4] M. El-Sherif, L. Bansal, and J. Yuan, "Fiber optic sensors for detection of toxic and biological threats," Sensors, vol. 7, no. 12, pp. 3100-3118, 2007.

[5] A. Leung, P. M. Shankar, and R. Mutharasan, "A review of fiberoptic biosensors," Sensors and Actuators, B: Chemical, vol. 125, no. 2, pp. 688-703, 2007.

[6] M. Yang and J. Dai, "Review on optical fiber sensors with sensitive thin films?" Photonic Sensors, vol. 2, no. 1, pp. 14-28, 2012.

[7] B. D. Gupta and R. K. Verma, "Surface plasmon resonancebased fiber optic sensors: principle, probe designs, and some applications," Journal of Sensors, vol. 2009, Article ID 979761, 12 pages, 2009.

[8] B. Troia, A. Paolicelli, F. de Leonardis, and V. M. N. Passaro, "Photonic crystals for optical sensing: a review," in Advances in Photonic Crystals, V. M. N. Passaro, Ed., chapter 11, pp. 241-295, InTech Publisher, 2013.

[9] A. M. Pinto and M. Lopez-Amo, "Photonic crystal fibers for sensing applications," Journal of Sensors, vol. 2012, Article ID 598178, 21 pages, 2012.

[10] M. Skorobogatiy, "Microstructured and photonic bandgap fibers for applications in the resonant bio- and chemical sensors," Journal of Sensors, vol. 2009, Article ID 524237, 20 pages, 2009.

[11] H. Qu and M. Skorobogatiy, "Resonant bio- and chemical sensors using low-refractive-index-contrast liquid-core Bragg fibers," Sensors and Actuators B: Chemical, vol. 161, no. 1, pp. 261268, 2012.

[12] H. Qu, B. Ung, M. Roze, and M. Skorobogatiy, "All photonic bandgap fiber spectroscopic system for detection of refractive index changes in aqueous analytes," Sensors and Actuators B: Chemical, vol. 161, no. 1, pp. 235-243, 2012.

[13] H. Qu, B. Ung, and M. Skorobogatiy, "Liquid filled hollow core photonic bandgap fiber sensor," in Proceedings of the Conference on Optical Sensors, Toronto, Canada, June 2011.

[14] H. Qu, T. Brastaviceanu, F. Bergeron, J. Olesik, I. Pavlov, and M. Skorobogatiy, "Micro-displacement sensors based on plastic photonic bandgap bragg fibers," in Proceedings of the Frontiers in Optics (FIO '13), Orlando, Fla, USA, October 2013.

[15] V. Matějec, I. Kašík, O. Podrazký et al., "Preparation and characterization of bragg fibers for delivery of laser radiation at 1064 nm," Radioengineering, vol. 22, no. 1, pp. 346-351, 2013.

[16] V. Matejec, I. Kasik, O. Podrazky et al., "Preparation and characterization of Bragg fibers with air cores for transfer of laser radiation," in 2nd Micro-structured and Specialty Optical Fibres, K. Kalli, J. Kanka, and A. Mendez, Eds., vol. 8775 of Proceedings of SPIE, p. 877508, Prague, Czech Republic, May 2013. 
[17] P. Yeh, A. Yariv, and C. Hong, "Electromagnetic propagation in periodic stratified media. I. General theory," Journal of the Optical Society of America, vol. 67, no. 4, pp. 423-438, 1977.

[18] A. Song, S. Parus, and R. Kopelman, "High-performance fiber optic $\mathrm{pH}$ microsensors for practical physiological measurements using a dual-emission sensitive dye," Analytical Chemistry, vol. 69, no. 5, pp. 863-867, 1997.

[19] http://www.presens.de/products/category/category/sensorprobes.html.

[20] T. Martan, J. Aubrecht, O. Podrazký, V. Matějec, and I. Kašík, "Detection of hydrocarbons using suspended core microstructured optical fiber," Sensors and Actuators B: Chemical, vol. 202, pp. 123-128, 2014. 

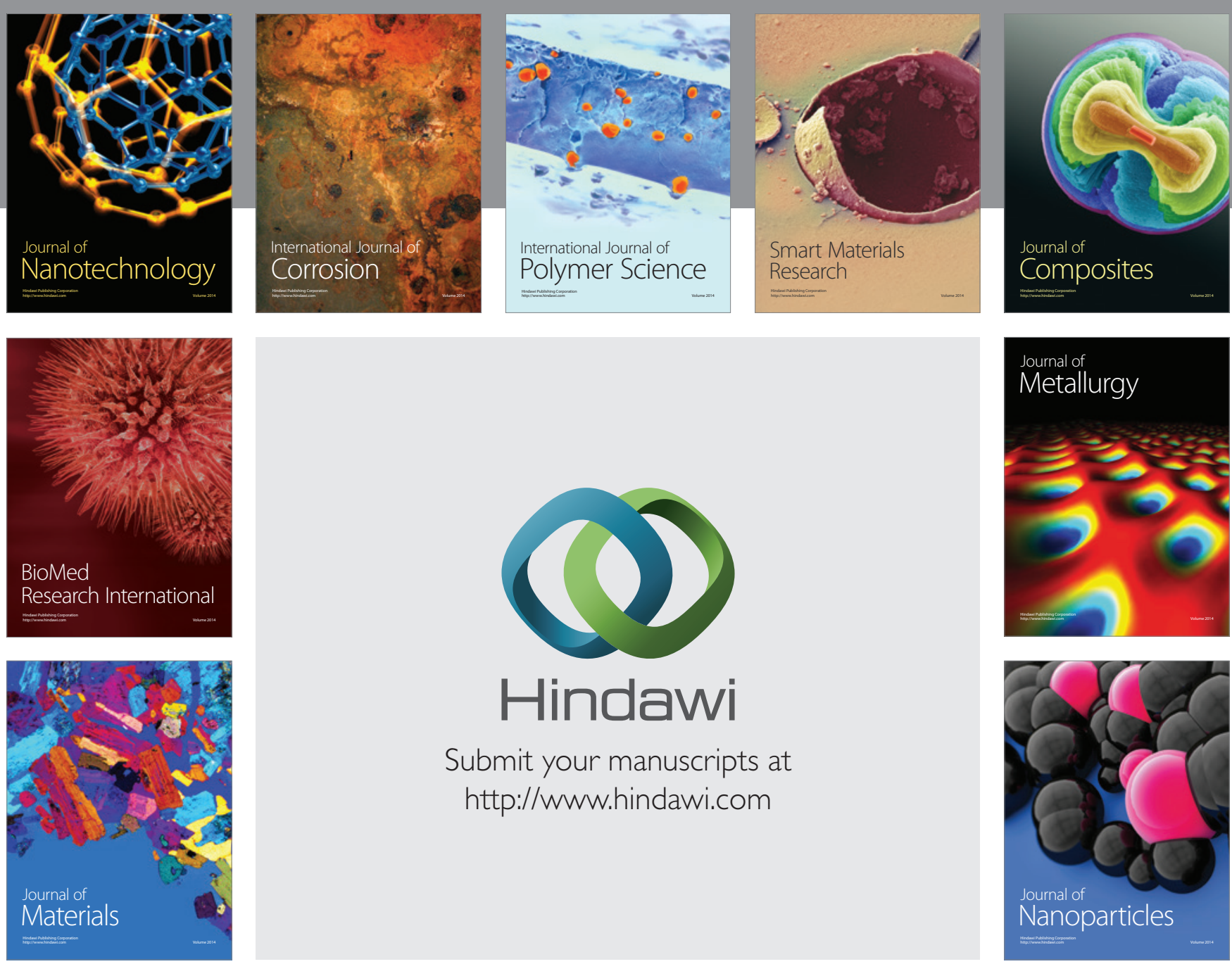

Submit your manuscripts at http://www.hindawi.com
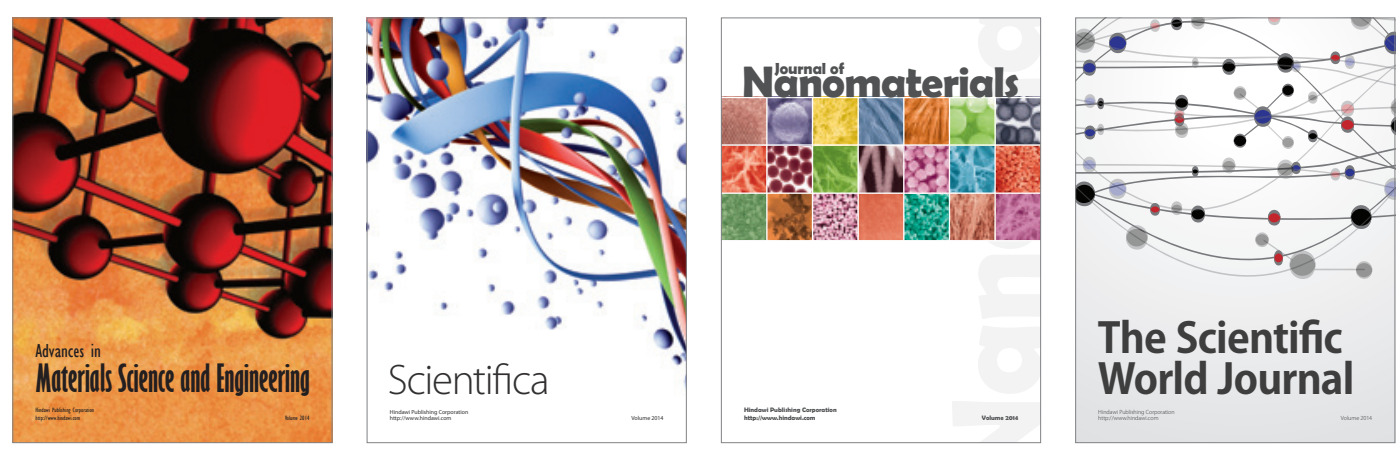

\section{The Scientific World Journal}
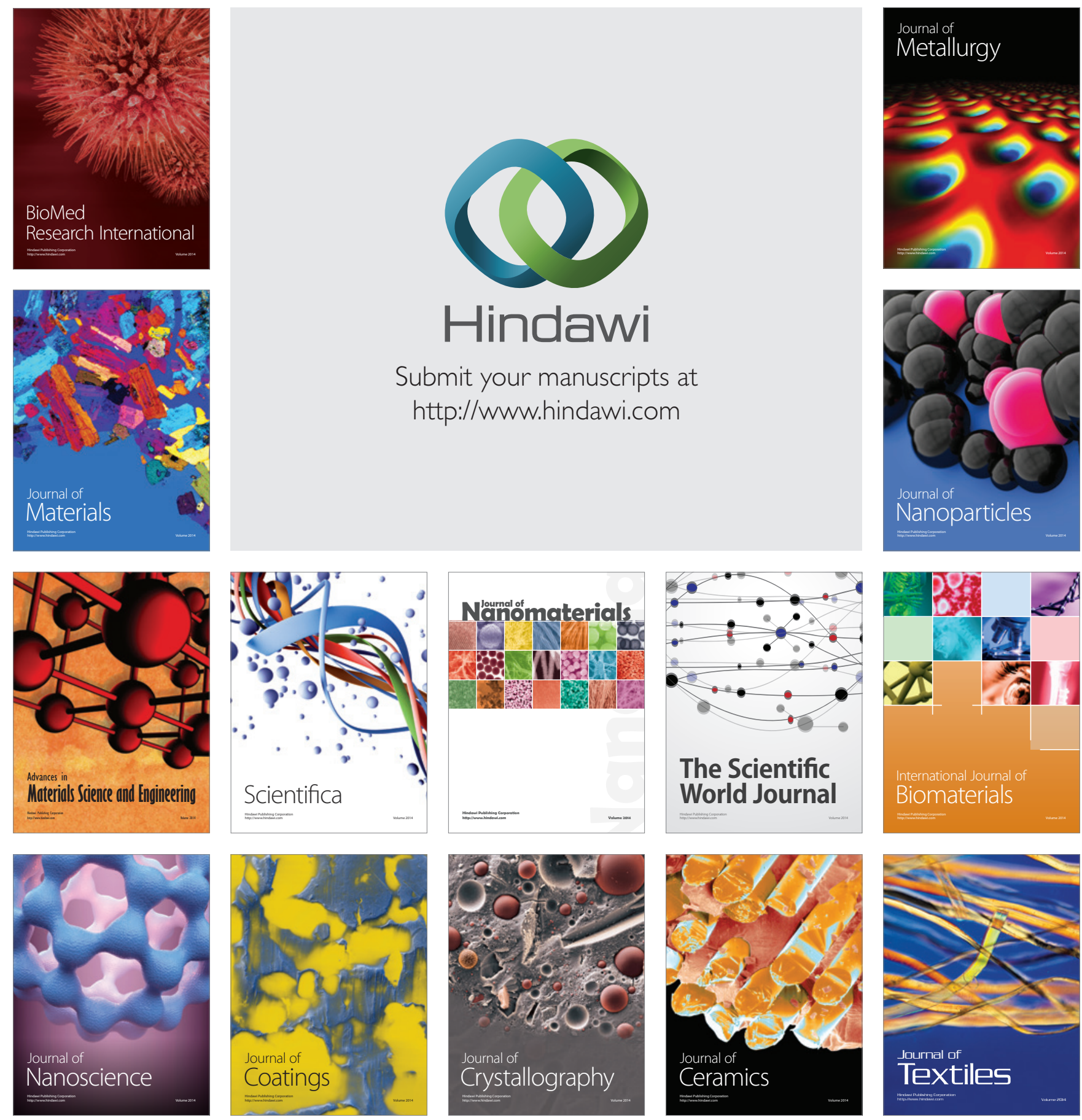